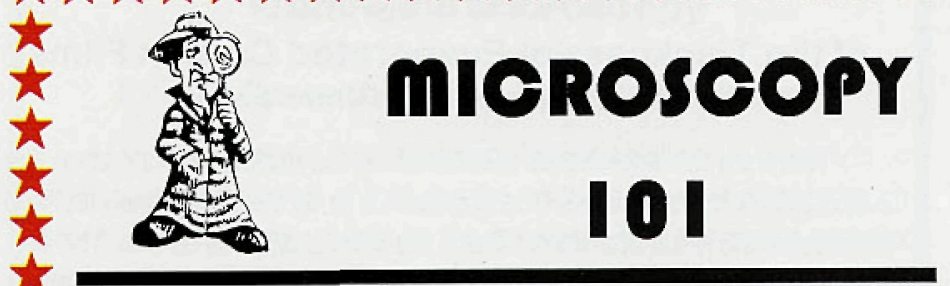

We appreciate the response to this publication feature - and weicome all contributions. Contributions may be sent to Phil Oshel, our Technical Editor at:
Mr. Phil Oshel
(608) $833-2885$
PO Box 620068
eMail: oshel@terracom.net
Middleton WI 53562

\section{Bands in SEM Images}

We used to get banding in the images of our Hitachi S-800 FESEM, in both backscattered and secondary electron photographed images. This drove us nuts for many long months! Finally I realized that it really only appeared on the recording CRT, and not on the visual CRTs. I hauled out a really old SEM manual* that dealt with all kinds of weirdness. It showed banding on a CRT that was a result of dirty or deficient high voltage to the CRT. I gingerly cleaned the high voltage contacts to the CRT and the problem disappeared. It reappears every few years. and I imagine it's our humid and somewhat volcanic air that corrodes these contacts. Cleaning them up works. Hitachi remains dubious about this, by the way.

The high voltage supply to the CRT is $10 \mathrm{kV}$ ! Be careful! The cleaning I have done has so far not involved actually polishing contacts, but merely blowing compressed clean, dry air over the contacts, making sure they are seated, cleaning the surrounding areas, and carefully closing everything up again. There are a number of circuits in most instruments that are still energized or retain a charge when the instrument is off or even disconnected.

If the bands appear on digital images, then the source of the signal still needs to be tracked down and examined for weak contacts.

* Combing the manual for clues, I decided it may have been put out by JEOL. The spellings are British, it describes itself as a "booklet, and it is in $8^{\prime \prime} \times 10^{\prime \prime}$ format. The terminology and references to parts of the instrument make it seem to be from ca. 1980 ? Maybe before, or as late as 1985. It's entitled A Guide to Scanning Microscope Observation. It has some great tips for figuring out what's going on based on what kind of image one gets. I've never seen another book that addresses problems from such a basic, practical viewpoint. Most books are full of schema and formulae.

My copy begins on page 2 and has no cover. If anyone recognizes this book, please send the complete reference to the Microscopy Today Technical Editor [Philip Oshel, oshel@terracom.net, or post the information on the microscopy list server.

Tina (Weatherby) Carvalho, University of Hawail at Manoa

\section{A SEM Tip for Examining Mixtures of Mineral and Organic Particles}

The usual practice is to mount the sample in a quick-setting epoxy resin, with most of the sample in the bottom. I use silicon rubber cups with a one inch inside diameter, about $1 / 2$ inch deep. Mix particles and resin together, about 50/50, put that in the bottom of the cup, then fill with clear resin. The set resin is then ground on a graded series of sandpaper disks with water running on them, then polished on 5 micron diamond suspension to cross-section the particles. The sample is then gold or carbon coated.

Organic or polymer particles and minerals are easily separated by backscattered imaging (BSE). The organics, being composed of light elements, will show darker than the mineral, which is composed of heavier elements. I usually use a $20 \mathrm{kV}$ e-beam for BSE imaging, then vary, the condenser lens to adjust the contrast and illuminate the phases l'm interested in. You can also ty adjusting the $\mathrm{KV}$ of the beam to light up the phases of interest. A photo of secondary electrons and BSE of the same area is often helpful.

Particle size measurements may be better on a sample of grains sprinkled on a sticky tab and imaged by BSE, but topographical interference, i.e. shadows, may degrade the analysis

Mary Mager, University of British Columbia

\section{Some Sources of Flexible Needles for Handling Microscopy Specimens}

As an electron microscopist who has come from a neurophysiology background, I have used various fine needles for "dusting" off debris. You need to find the one that feels right.

Cat whiskers are long, pointy, strong, and flexible. They are particularly good for chasing tiny bubbles out of microelectrodes or capillary tubes.

Finely drawn glass: Heat a pipette or rod over a Bunsen burner and draw it out until it breaks. Find somewhere along the long string that has the right size and flexibility, but won't break and leave more debris!

Cactus spines: They come in many shapes and sizes. Also useful for pinning down things for dissection that can't come into contact with metal.

Find someone in neurobiology who does microelectrode recording and get them to make some electrodes, which are capillary tubes drawn to a very fine point. You can probably get some in the micron range. Beveled, even!

Insect Minuten pins mounted on a stick are very strong, but may scratch your substrate. They can be ground down for a finer point.

Eyelashes, beard hair, and other body hairs each have different properties. Have fun experimenting.

Tina (Weatherby) Carvaiho, University of Hawaii at Manoa

\section{Supravital Stain for blood}

Paul Ehrlich pioneered the air-dried blood smear before the turn of the century. Air-dried, because wet fixatives washed the specimen off of the slide. Various stains were tried at first, but eventually the Romanowski stains (Wright's Giemsa, etc.) prevailed. When a smear is air-dried, the spherical white cells flatten out to become discs, introducing a number of artifacts, notably loss of nucleolar staining.

For some artifactual reason, nucleolar staining is retained in many leukemic cells, leading to the term "blast". However, with wet fixation or with supravital staining of live cells, even normal, mature small lymphocytes contain nucleoli.

So information obtainable from blood staining depends more on the mode of fixation than on the stain

Wet fixation of a blood clot is almost useless because of the overwhelming preponderance of red blood cells. Wet fixation of a buffy coat is possible if it is treated as a cell block, but centrifugation introduces a few relatively minor artifacts of its own. Any specimen that is embedded in paraffin undergoes considerable shrinkage during alcoholic dehydration.

Supravital staining means putting a small drop of blood on a stained slide 